\title{
Extinction performance as a function of incentive magnitude and number of acquisition trials'
}

\author{
James R. Ison and Patriek E. Cook \\ UNIVERSITY OF ROCHESTER
}

\begin{abstract}
Abstraet
Food deprived rats received straight runway training with either a small or large incentive (1 or 10 food pellets) for either 76 or 31 acquisition trials, followed by 30 extinction trials. At the large incentive the group given the greater number of trials exhibited less response strength in extinction whereas at the small incentive the reverse was observed. Additionally both groups given the large incentive demonstrated a more rapid decrement in extinction than their respective small magnitude groups.
\end{abstract}

\section{Introduction}

It has been suggested (Ison, 1962) that in the straight runway the relationship between extinction performance and number of reinforcements depends upon the magnitude of the incentive, such that at a high magnitude increased numbers of reinforcements lead to less extinction response strength whereas at a low magnitude they result in increased extinction response strength. The present experiment lends some support to this proposed interaction.

\section{Method}

Fifty-six hooded rats, 30 males and 26 females, ranging in age from 100 to 120 days, were obtained from Rockland Farms, New City, New York. They were randomly assigned to the cells $(n, 14)$ of the $2 \times 2$ factorial design resulting from two levels of acquisition trials (76 and 31 ) and incentive magnitudes (10 or 1 Formula A .037 milligram Noyes food pellets), with approximately the same distribution of males and females in each group. One female $\mathrm{S}$ was discarded because of failure to eat in acquisition.

The straight alleyway consisted of a 12 inch startbox and a 40 inch runway, both constructed of unpainted grey sheetmetal, and a 10 inch black wooden goalbox. The three sections were covered with frosted plexiglass. The start box and runway were 4 inches high and 3 inches wide and the goal box was 4 inches high and 5 inches wide. A black wooden partition, 1 inch high and 5 inches wide, placed 2 inches from the end of the goal box, shielded a clear glasss foodcup. Frosted plexiglass guillotine doors were placed at the exit of the start box and the entrance to the goal box. The start box door was counterweighted and a full 4 inch excursion took .31 seconds (SD, .01 seconds) independent of E's response speed on the release cable. A photocell placed 6 inches from the start door and a second placed 1 inch inside the goal box, in conjunction with a microswitch on the start door, operated two clocks to record start and running times which were then converted to speeds.
The Ss were placed on a food deprivation schedule for seven days prior to runway training, on each of which they received 12 grams of food powder in wet mash in a white cup 15 minutes after the daily treatment. During training this amount was reduced by the amount of food received in the runway. Pre-training consisted of three days of gentling, 3-5 minutes per day, and four subsequent days on each of which $\mathrm{S}$ was given a single presentation of the appropriate reinforcement in an unpainted wooden box. On the eighth day two groups began five days of acquisition training (15 trials per day) with 10 pellets reward or 1 pellet, and two groups began three days of handling trials (15 per day) in which $S$ was taken from the detention cage to the apparatus and then replaced in the cage. The two latter groups then received two days of acquisition training at 10 pellets reward or 1 pellet. Two days (30 trials) of extinction training followed. An empty glass foodcup was always present in the goal box wherein $\mathrm{S}$ was detained for 10 seconds. The first day of extinction included an additional initial reinforcement. Water was always available during the intertrial intervals, which were 4-5 minutes long. If an extinction response time on either clock exceeded 120 seconds $\mathrm{S}$ was taken from the apparatus and placed in the goal box.

\section{Hesults}

Starting and running speeds throughout the experiment are presented in Fig. 1. Acquisition was generally unremarkable except for the small effect of the acquisition variables on running speeds. Analysis of variance for the final day of acquisition indicated that both higher magnitude and more trials led to faster starting speeds ( $\mathrm{p}<$ .05) with no interaction, but neither variable had a significant effect on running speeds. This lack of significance is probably due to a differential within day satiation resulting from the consumption of the large reward, a hypothesis that is supported by the typical within day convergence of the two magnitudes. It should be noted that this possible satiation effect could not directly influence extinction performance because neither set of groups was reinforced in extinction.

On the first day of extinction the proposed interaction was obtained in the four groups. Analyses of variance of these data, using extinction trials blocked in sets of five as a "within" variable, yielded a significant Magnitude $x$ Acquisition Trials interaction for starting speed ( $F$, 6.77 ; df, $1 / 50 ; p<.025)$ but not for running speed $(F$, $1.89 ; \mathrm{df}, 1 / 50 ; \mathrm{p}<.10)$; for running speed the main effect of magnitude was significant $(\mathrm{F}, 4.96 ; \mathrm{df}, 1 / 50 ; \mathrm{p}<.05)$. Two t-tests, using the MSE (b) from the preceding analysis of variance, indicated that at the large incentive the 


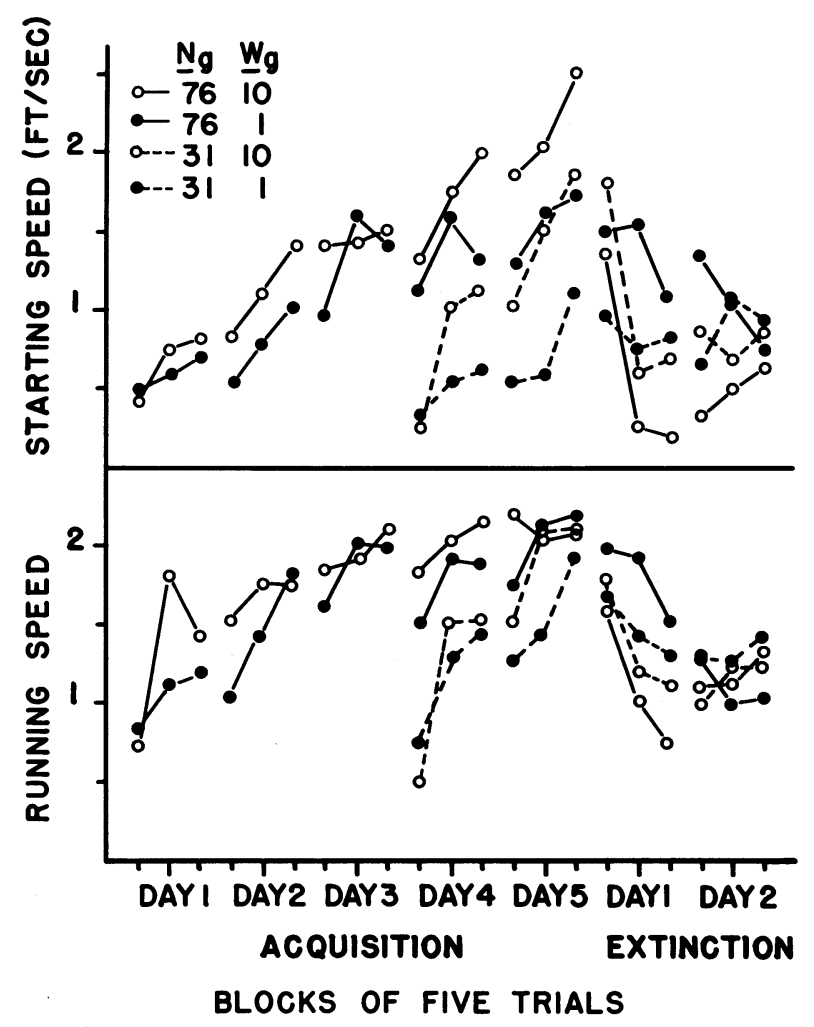

Fig. 1. Starting speed and running speed in acquisition and extinction as a function of two levels of incentive magnitude (Wg; 10 or 1 ) and two levels of number of acquisition trials ( $\mathrm{Ng} ; 76$ or 31 ).

group given the greater number of trials had a lower mean starting speed $(t(50), 6.88 ; \mathrm{p}<.01)$ whereas at the small incentive the reverse was true $(t(50), 7.22 ; p<$ $.01)$. A further effect of some interest was the interaction between magnitude and number of extinction trials (for starting speed F, 6.36; df, 2/100; p <.005; for running speed F, 3.79; df, $2 / 100 ; p<.05)$. On each measure the high incentive groups demonstrated a precipitous decline in extinction whereas the low incentive groups had a more gradual decline. It should be noted that if extinction performance in Fig. 1 were redrawn as percent terminal acquisition performance the difference between the two low incentive groups would be largely eliminated whereas the difference between the two high incentive groups would be accentuated. This suggests that with the low incentive the major determiner of extinction performance is the terminal acquisition level but at the high incentive a further process is indicated.
The second day of extinction was marked by the convergence of the four groups from their first day differences. Neither acquisition variable had a reliable effect on the second extinction day.

\section{Diseussion}

It is apparent that the negative relationship between number of reinforcements and performance in extinction can be obtained more readily when the incentive used in acquisition is large. This was probably the critical factor in the different results obtained by Ison (1962), North \& Stimmel (1960) and Hill \& Spear (1963). In the first two experiments a large incentive was used and increased numbers of reinforcements resulted in less resistance to extinction whereas in the third experiment a small incentive was used and the opposite effect was found.

This does not necessarily mean that the negative relationship found here only with the large incentive could never be obtained with a small incentive. One might speculate, for example, that in the straight runway there is one general non-monotone function relating response strength in extinction to the number of prior reinforcements. Further, the maximum point of the function in turn is related to the magnitude of the incentive such that as the incentive increases the maximum would occur at a progressively smaller number of acquisition trials.

If extinction behavior were so complexly determined one might anticipate that the experimental literature would reveal a very confused picture; values chosen in some experiments would straddle the maximum and result in a lack of difference between groups, whereas others would produce reliable differences but in opposite directions. In fact the experimental work on extinction in the runway and in the related area of discrimination reversal demonstrates this pattern of discordant outcomes.

\section{References}

HILL, W. F., \& SPEAR, N. E. Extinction in a runway as a function of acquisition level and reinforcement percentage. J. exp. Psychol., 1963, 65, 495-500.

ISON, J. R. Resistance to extinction as a function of number of reinforcements. J. exp. Psychol., 1962, 64, 314-317.

NORTH, A. J., \& STIMMEL, D. T. Extinction of an instrumental response following a large number of reinforcements. Psychol. Rep., 1960, 6, 227-234.

\section{Note}

1. This research was supported by research grants MH07897 and NSF GB-1501 to the senior author, and was performed during the junior author's tenure as an NSF Undergraduate Research Participant. 